\title{
SIMPLICIAL QUANTUM GRAVITY WITH HIGHER DERIVATIVE TERMS: FORMALISM AND NUMERICAL RESULTS IN FOUR DIMENSIONS
}

\author{
Herbert W. HAMBER \\ Institute for Advanced Study, Princeton, NJ 08540, USA \\ Ruth M. WILLIAMS \\ Girton College and Department of Applied Mathematics and Theoretical Physics, \\ Cambridge, CB3 9EW, England \\ Received 16 September 1985
}

\begin{abstract}
Higher derivative terms for Regge's formulation of lattice gravity are discussed. The analytic weak-field expansion for the regular tessellation $\alpha_{5}$ of the four-sphere is presented. Preliminary numerical results for some computations in four dimensions are also discussed.
\end{abstract}

\section{Introduction}

The study of higher derivative gravity theories dates back to more than thirty years ago (for a historical review see ref. [1]). It has been known for some time that if one attempts to quantize the Einstein theory of gravity one encounters two major difficulties. The field equations for the metric are derived from an action that is unbounded from below, and the path integral is therefore mathematically ill-defined. Furthermore the coupling constant in Einstein gravity (Newton's constant) has dimension of inverse mass squared (in units $h=c=1$ ), and this leads to a nonrenormalizable quantum theory, as can be verified by doing explicit Feynman diagram perturbation theory [2-4].

One possible attitude is to hope that these problems will be cured in the context of a grand unified theory like supergravity. Alternatively, one might argue that the above problems hint at a fundamental incompatibility between gravity and quantum mechanics, and any modification of the Einstein action will in general lead to new undetermined parameters.

Of course the argument about naturalness and simplicity of the Einstein theory can be turned around, in the sense that a quantum theory of gravity should just provide the answer for why, starting with the most general microscopic theory consistent with general invariance principles, some terms appear in the low-energy 
effective lagrangian and others do not. The question then becomes of course a dynamical one. In other field theoretic contexts this phenomenon is connected with the flow of the coupling constants as the length scale at which the theory is probed is changed, and the concept of scaling dimensions and operator relevance [5].

Within the framework of continuum local field theories, the alternative possibility is thus to include in the action those terms that are generated by renormalization, and see whether the resulting action leads to a tractable and perhaps meaningful quantum theory [6]. It turns out that only two additional terms, involving fourth derivatives of the metric, need to be added to the Einstein action in order to obtain a perturbatively renormalizable theory, and cure at the same time the unboundedness problem [7]. More remarkably, the resulting theory is asymptotically free in the new coupling constants (if they are chosen with the right sign) and is thus ultraviolet stable $[8,9]$. Furthermore, because of the asymptotic freedom the infrared behavior of the theory is computable and cutoff-independent. This suggests that some class of higher derivative gravity theories with a cutoff can be defined, such that a truly cutoff independent continuum limit exists, and can be constructed using the renormalization group.

Unfortunately, as will be further discussed below, it appears that the theory has some potential problems with unitarity. If one looks at the tree-level graviton propagator one finds that it exhibits a massive ghost pole. It has been argued that radiative corrections restore the unitarity of the theory (by decoupling the ghosts), but it seems unlikely that these and other questions (like the recovery of a newtonian limit) can be answered within the context of weak coupling perturbation theory. In fact the complexity of the theory resembles quantum chromo-dynamics, for which other tools (like the lattice regularization and non-perturbative methods) are needed to control and understand the low-energy, large distance properties. It is in this spirit that one has decided to turn to a discrete formulation of quantum gravity. The lattice is introduced as an aid to formulating and calculating the theory in the same way that one uses finite differences both to define derivatives and to obtain approximate numerical solutions of differential equations. For recent reviews of Yang-Mills theories defined on a lattice see refs. [11].

Gravity on a lattice was in fact formulated some time ago by Regge [12]. In his work he showed that lattice gravity can be described by a simplicial net in which the elementary variables are the edge lengths connecting the points in the net. Here Regge's formulation and its extension to higher derivative gravity will be discussed in some detail. It is clear that if some class of higher derivative gravity theories is well defined and has a sensible low-energy limit, it should agree with the experimental evidence. This suggests that the effects of the higher derivative terms should vanish in the low-energy limit, and that the observed smallness of the cosmological constant be explained in a natural way as a consequence of renormalization effects. These are questions that presumably can best be answered by studying the non-perturbative dynamics of the theory on a space-time lattice. 
Consider the euclidean Einstein action without a cosmological constant term

$$
I_{\mathrm{E}}=-\frac{1}{16 \pi G} \int \mathrm{d}^{4} x \sqrt{\mathrm{g}} R,
$$

where $G$ is Newton's constant, $\sqrt{g}$ is the determinant of the metric $g_{\mu \nu}$, and $R$ is the scalar curvature. Here boundary terms have been dropped, and couplings to matter fields are not considered. Variation with respect to the metric leads to the classical equations of motion for the gravitational field in a vacuum

$$
\delta I_{\mathrm{E}}=0 \Rightarrow R_{\mu \nu}-\frac{1}{2} g_{\mu \nu} R=0
$$

If one attempts to write down a path integral of the form

$$
Z=\int_{\text {geometries }} \mathrm{e}^{-I_{\mathrm{E}}}
$$

(which will in general depend on a specified initial and final three-geometry) one soon realizes that it appears ill defined due to the fact that the scalar curvature can become arbitrarily positive (or negative). This in turn is a consequence of the fact that while gravitational radiation has positive energy, gravitational potential energy is negative because gravity is attractive. The gravitational action is unbounded from below and the functional integral strongly depends on how the unboundedness is cutoff [2]. To see more clearly that the gravitational action can be made arbitrarily negative consider a conformal transformation $\tilde{g}_{\mu \nu}=\Omega^{2} g_{\mu \nu}$ where $\Omega$ is a positive function. Then the Einstein action transforms into

$$
I_{\mathrm{E}}(\tilde{g})=-\frac{1}{16 \pi G} \int \mathrm{d}^{4} x \sqrt{g}\left(\Omega^{2} R+6 g^{\mu \nu} \partial_{\mu} \Omega \partial_{\nu} \Omega\right),
$$

which can be made arbitrarily negative by choosing a rapidly varying conformal factor $\Omega$.

A second serious problem is connected with the fact that the coupling constant $G^{-1}$ has dimension of mass to the power $(d-2)$ in $d$ space-time dimensions and suggests that the theory is not perturbatively renormalizable above two dimensions. It has been shown that close to four dimensions in order to renormalize the theory at one loop one needs to introduce higher derivative counterterms, which are needed to cancel the divergences proportional (in dimensional regularization) to [3]

$$
\Delta I=\frac{1}{8 \pi^{2}(d-4)} \int \mathrm{d}^{4} x \sqrt{\mathrm{g}}\left[\frac{1}{240} R_{\mu \nu \rho \sigma} R^{\mu \nu \rho \sigma}+\frac{1}{80} R^{2}\right] .
$$


A possible solution to the unboundedness problem has been described by Hawking [2], who suggests performing the integration over all metrics by first integrating over conformal factors distorting the integration contour in the complex plane to avoid the unboundedness problem, followed by an integration over conformal equivalence classes of metrics.

A second possibility is to add to the Einstein action extra terms, including higher derivative ones like $R^{2}$, in a carefully chosen combination which makes the total action bounded from below. It turns out that only up to fourth derivative terms need to be considered in order to cure the renormalizability problem. Thus one is led to consider the extended gravitational action [9]

$$
I=\int \mathrm{d}^{4} x \sqrt{g}\left[\lambda-k R+\frac{1}{4} a R_{\mu \nu \rho \sigma} R^{\mu \nu \rho \sigma}+\frac{1}{3}\left(b-\frac{1}{4} a\right) R^{2}\right],
$$

with a cosmological constant term (proportional to $\lambda$ ), the Einstein term ( $k=$ $1 / \mathbf{1 6 \pi} G$ where $G$ is the bare Newton constant), and two higher derivative terms with additional dimensionless coupling constants $a^{-1}$ and $b^{-1}$. Even though there are four possible higher derivative terms in four dimensions which do not give rise to topological invariants (proportional to integrals of $C_{\mu \nu \rho \sigma}^{2}, R_{\mu \nu \rho \sigma}^{2}, R_{\mu \nu}^{2}$ and $R^{2}$ ), only two are found to be independent for a manifold of fixed topology, if one uses some identities for the Riemann tensor and the integral expression for the Euler characteristic [13].

The higher derivative action of eq. (1.6) was shown to be renormalizable to all orders in perturbation theory [7]. Perturbation theory is usually performed around flat space, which requires $\lambda=0$. One sets $g_{\mu \nu}=\eta_{\mu \nu}+h_{\mu \nu}$ and expands the action in powers of $h_{\mu \nu}$. (If $\lambda$ is nonzero, one has to expand around a solution of the classical equations of motion for higher derivative gravity $[1,14]$ with a $\lambda$ term, and the solution will no longer be constant over space-time). The quadratic part of the action is [9]

$$
\begin{aligned}
I_{\text {linearized }}=\frac{1}{2} \int \mathrm{d}^{4} x[ & h_{\mu \nu}\left\{\frac{1}{2} k+\frac{1}{2} a(-\Delta)\right\}(-\Delta) P_{\mu \nu \rho \sigma}^{(2)} h_{\rho \sigma} \\
& \left.+h_{\mu \nu}\{-k+2 b(-\Delta)\}(-\Delta) P_{\mu \nu \rho \sigma}^{(0)} h_{\rho \sigma}\right],
\end{aligned}
$$

where $\Delta$ is the $4 \mathrm{~d}$ laplacian and $P^{(2)}$ and $P^{(0)}$ are projection operators for the spin-two and spin-zero modes, respectively, of the linearized gravitational field. Let us recall that the three relevant projection operators are given in momentum 
space by

$$
\begin{aligned}
P_{\mu \nu \alpha \beta}^{(2)}= & \frac{1}{3}\left(\frac{k_{\mu} k_{\nu}}{k^{2}} \delta_{\alpha \beta}+\frac{k_{\alpha} k_{\beta}}{k^{2}} \delta_{\mu \nu}\right) \\
& -\frac{1}{2} \frac{1}{k^{2}}\left(k_{\mu} k_{\alpha} \delta_{\nu \beta}+k_{\mu} k_{\beta} \delta_{\nu \alpha}+k_{\nu} k_{\alpha} \delta_{\mu \beta}+k_{\nu} k_{\beta} \delta_{\mu \alpha}\right) \\
& +\frac{1}{2}\left(\delta_{\mu \alpha} \delta_{\nu \beta}+\delta_{\mu \beta} \delta_{\nu \alpha}\right)-\frac{1}{3} \delta_{\mu \nu} \delta_{\alpha \beta}+\frac{2}{3} \frac{1}{k^{4}} k_{\mu} k_{\nu} k_{\alpha} k_{\beta}, \\
P_{\mu \nu \alpha \beta}^{(1)}= & \frac{1}{2} \frac{1}{k^{2}}\left(k_{\mu} k_{\alpha} \delta_{\nu \beta}=k_{\mu} k_{\beta} \delta_{\nu \alpha}+k_{\nu} k_{\alpha} \delta_{\mu \beta}+k_{\nu} k_{\beta} \delta_{\mu \alpha}\right)-\frac{1}{k^{4}} k_{\mu} k_{\nu} k_{\alpha} k_{\beta}, \\
P_{\mu \nu \alpha \beta}^{(0)}= & -\frac{1}{3}\left(\frac{k_{\mu} k_{\nu}}{k^{2}} \delta_{\alpha \beta}+\frac{k_{\alpha} k_{\beta}}{k^{2}} \delta_{\mu \nu}\right)+\frac{1}{3} \delta_{\mu \nu} \delta_{\alpha \beta}+\frac{1}{3} \frac{1}{k^{4}} k_{\mu} k_{\nu} k_{\alpha} k_{\beta}
\end{aligned}
$$

and add up to give the unit matrix

$$
\sum_{i} P_{\mu \nu \alpha \beta}^{(i)}=I_{\mu \nu \alpha \beta}=\frac{1}{2}\left(\delta_{\mu \nu} \delta_{\alpha \beta}-\delta_{\nu \alpha} \delta_{\mu \beta}\right) .
$$

As can be seen, the spin-zero mode is responsible for the unboundedness of the pure Einstein action since its kinetic term comes with the wrong sign. (In the linearized action it appears as a ghost contribution).

Including radiative corrections to one-loop order one finds that the theory is asymptotically free in the couplings $a$ and $b$. To this order (small $a^{-1}, b^{-1}$ ) the renormalization group equations for the two higher derivative couplings and the dimensionless cosmological constant $\bar{\lambda}=a \lambda / k^{2}$ computed in ref. [9] are

$$
\begin{gathered}
\frac{\partial a}{\partial \ln L}=\beta_{a}=\frac{1}{16 \pi^{2}} \frac{133}{10}+\cdots, \\
a \frac{\partial(b / a)}{\partial \ln L}=\beta_{b}-\frac{b}{a} \beta_{a}=\frac{1}{16 \pi^{2}}\left[\frac{10 b^{2}}{3 a^{2}}-\frac{183 b}{10 a}-\frac{1}{12}\right]+\cdots, \\
a \frac{\partial \bar{\lambda}}{\partial \ln L}=\frac{1}{16 \pi^{2}}\left[-\frac{20 b}{3 a}+\frac{a}{6 b}+\frac{183}{10}\right] \bar{\lambda}+\frac{5}{4}+\frac{a^{2}}{16 b^{2}}+\cdots,
\end{gathered}
$$

where $L$ is the cutoff in momentum space. The first equation gives an ultraviolet fixed point at $a^{-1}=0$. The second one shows that there is an ultraviolet fixed point at $b / a=\frac{1}{200}(549-\sqrt{302401}) \approx-0.0046$. The choice of $a++$ sign for the root gives on the other hand an infrared fixed point at $b / a \approx+5.4946$. Therefore also the 
coupling $b$ is asymptotically free, under the assumption $b<0$. The theory with the Weyl $C^{2}$ term alone $(b=0)$ is conformally invariant, but not perturbatively renormalizable because of the conformal anomaly [9]. Due to the existence of the infrared fixed point in $b / a$, the renormalized dimensionless cosmological constant $\lambda / k^{2}$ can be driven to zero in the infrared, if it is sufficiently small to start with. This conclusion is of course only true if the quartic and quadratic divergences in $\lambda$ and $k$ are shown to vanish to all orders. This point will be discussed further below.

In order to see the problems with unitarity consider the graviton propagator for higher derivative gravity in the weak field limit. In momentum space the free propagator for $\lambda=0$ can be written as

$$
\begin{aligned}
\frac{1}{k}\left\langle h_{\mu \nu}(q) h_{\rho \sigma}(-q)\right\rangle= & \frac{2 P_{\mu \nu \rho \sigma}^{(2)}}{q^{2}+(a / k) q^{4}}+\frac{P_{\mu \nu \rho \sigma}^{(0)}}{-q^{2}+(2 b / k) q^{4}}+\text { gauge terms } \\
= & 2 P_{\mu \nu \rho \sigma}^{(2)}\left[\frac{1}{q^{2}}-\frac{1}{q^{2}+k / a}\right]+P_{\mu \nu \rho \sigma}^{(0)}\left[-\frac{1}{q^{2}}+\frac{1}{q^{2}-k / 2 b}\right] \\
& + \text { gauge terms. }
\end{aligned}
$$

The higher derivative terms improve the ultraviolet behavior of the theory since the propagator now falls off as $1 / q^{4}$ for large $q^{2}$. On the other hand the theory has a spin-two ghost of bare mass $m_{2}=\mu / \sqrt{a}$ and a spin-zero particle of bare mass $m_{0}=\mu / \sqrt{-2 b}$ where $\mu=\sqrt{k}$ is the bare Planck mass. We can set $\mu=\mu_{0} L$, where $\mu_{0}$ is a dimensionless coupling and $L$ is the ultraviolet cutoff in momentum space. Thus the tree level unitarity violating corrections are of order $q^{2} / L^{2}$.

The presence of massive states in the tree-level graviton propagator indicates also short distance deviations from the static newtonian potential, which in higher derivative gravity (in the weak field limit) has the form

$$
h_{00} \sim \frac{1}{\mu}\left[\frac{1}{r}-\frac{4}{3} \frac{\mathrm{e}^{-m_{2} r}}{r}+\frac{1}{3} \frac{\mathrm{e}^{-m_{0} r}}{r}\right] .
$$

In the absence of the Einstein term $(k=0)$ the potential is linear in $r$ and the theory is strongly infrared divergent, and it is not completely clear whether weak coupling perturbation theory around the tree-level solution is really trustworthy. The masses that give the potentially dangerous exponential corrections to the $1 / r$ behavior are of course bare masses, and the full renormalized gauge invariant part of the potential should be computed nonperturbatively (as in QCD) before any meaningful comparison with experiment is attempted. In fact the one-loop result for the running coupling $a$ ( $\beta_{a}$ is the one-loop coefficient of the beta function for the coupling $a$ )

$$
a^{-1}(r)=\frac{a^{-1}\left(r_{0}\right)}{1+a^{-1}\left(r_{0}\right) \beta_{a} \ln \left(r_{0} / r\right)}
$$


suggests strongly that the asymptotic freedom (running) coupling constants grow indefinitely in the infrared regime, and the massive ghost becomes increasingly heavy as $r_{0} / r$ goes to zero (low-energy limit), and possibly decouples completely

$$
\lim _{r / r_{0} \rightarrow \infty} m_{2}(r)=\frac{\mu}{\sqrt{a\left(r_{0}\right)}}\left(1+a^{-1}\left(r_{0}\right) \beta_{a} \ln \frac{r}{r_{0}}\right)^{1 / 2}=\infty .
$$

Here $r_{0}$ is the cutoff scale, not to be confused with the inverse Planck mass. The same reasoning can be applied to the massive spin-zero state.

These considerations are in fact far from being rigorous, but one should keep in mind that the one-loop result is qualitatively correct in $\lambda \phi^{4}$ and Yang-Mills theories in four dimensions. Also one should notice the fact that the graviton propagator as defined above is not gauge invariant. This is analogous to the gluon propagator in QCD: the perturbative massless gluons are in fact confined in massive glueballs and there is no gluon state in the physical gauge invariant spectrum. (The situation in gravity has of course to be different to some extent, since massless gravitons presumably do exist.) Therefore the real question to answer is whether the massive ádditional states contribute to gauge invariant correlation functions in the low-energy, large distance limit, in a way which is consistent with present experimental evidence. It is well known that weak coupling perturbation theory is useless in this regime for an asymptotically free theory.

Another potential problem is connected with the cosmological constant $\lambda$, whose value is observed to be of the order of $10^{-122}$ or less, in units of the Planck mass. (Experimentally one has $1 / \sqrt{G}=1.2 \times 10^{19} \mathrm{GeV}$ and $\lambda<(0.003 \mathrm{eV})^{4}$.) In higher derivative gravity one would expect purely on dimensional grounds a quartic divergence

$$
\lambda_{\mathrm{R}}=\lambda_{0}+c_{4} L^{4}+c_{2} L^{2}+\mathrm{O}(\ln L)
$$

( $L \sim r_{0}^{-1}$ is the ultraviolet cutoff), which has then to be canceled by fine-tuning the bare cosmological constant to one part in $10^{122}$, a rather unnatural procedure.

The procedure of setting all quartic and quadratic divergences equal to zero, as in dimensional regularization [3], seems somewhat formal and ad hoc, and clearly does not provide a physical explanation. On the other hand it has been argued that the quartic divergence for $\lambda$ is absent to all orders in perturbation theory $\left(c_{4}=0\right)$ for an appropriate choice of measure for the $g_{\mu \nu}$ fields [9]. The unique local gauge invariant measure is

$$
\mathrm{d} u(g)=\prod_{x} g^{-(d+1) / 2} \prod_{\mu \geqslant \nu} \mathrm{d} g_{\mu \nu}
$$


which is also scale invariant [15]. It is known that the measure can play a delicate role in canceling some spurious divergences in loop diagrams, that arise when a continuous symmetry is explicitly broken. An interesting point of view on the relevance of the measure in the quantum theory is presented in the second of refs. [4]. There it is argued that ambiguities in the definition of the measure reflect the lack of a unique definition for the metric tensor at short distances.

For completeness we list here the quadratic one-loop divergences [9] (quartic divergences are zero for the scale invariant measure)

$$
\begin{aligned}
& \lambda_{\mathrm{R}}=\lambda_{0}+\frac{k_{0}}{2 a}\left(5-\frac{a}{2 b}\right) \frac{L^{2}}{16 \pi^{2}}+\mathrm{O}(\ln L), \\
& k_{\mathrm{R}}=k_{0}+\frac{1}{2}\left(\frac{10 b}{3 a}-5\right) \frac{L^{2}}{16 \pi^{2}}+\mathrm{O}(\ln L),
\end{aligned}
$$

where the subscript $R$ denotes renormalized quantities and 0 bare ones. It appears surprising that these divergences are present, since the scale invariance of the measure suggests that the formal functional integral for pure gravity can only depend on the dimensionless couplings $\lambda / k^{2}, a$ and $b$.

The need for non-perturbative methods in the further study of higher derivative gravity leads us to study its formulation on a simplicial lattice, using Regge's "coordinate-independent description of general relativity". The relevant features of Regge calculus, including its representation of the Einstein term, are described in refs. [16] and [17], where the appropriate forms for the cosmological constant term and the simplest higher derivative term are derived. In this paper, concentrating on four dimensions, we shall discuss in detail other possible higher derivative terms in sect. 2. In sect. 3 we shall describe some analytic computations of the weak field expansion for the regular tessellation $\alpha_{5}$ of the four-sphere. In sect. 4 we shall describe our procedure for numerical computations in quantum gravity and present in sect. 5 some preliminary results on small lattices. Sect. 6 contains some concluding remarks and suggestions for further study.

\section{The representation of higher derivative terms on a simplicial lattice}

For the reasons described above, we wish to add to the Einstein action, with a possible cosmological constant term,

$$
I=\int \mathrm{d}^{4} x \sqrt{g}[\lambda-k R],
$$


a term or terms which are quadratic in the curvature. There are six such possibilities

$$
\begin{aligned}
& \int \mathrm{d}^{4} x \sqrt{g} R^{2}, \\
& \int \mathrm{d}^{4} x \sqrt{g} R_{\mu \nu} R^{\mu \nu}, \\
& \int \mathrm{d}^{4} x \sqrt{g} R_{\mu \nu \lambda \sigma} R^{\mu \nu \lambda \sigma}, \\
& \int \mathrm{d}^{4} x \sqrt{g} C_{\mu \nu \lambda \sigma} C^{\mu \nu \lambda \sigma}, \\
& \int \mathrm{d}^{4} x \sqrt{g} \varepsilon^{\mu \nu \kappa \lambda} \varepsilon^{\rho \sigma \omega \tau} R_{\mu \nu \rho \sigma} R_{\kappa \lambda \omega \tau}=128 \pi^{2} \chi, \\
& \int \mathrm{d}^{4} x \sqrt{g} \varepsilon^{\rho \sigma \kappa \lambda} R_{\mu \nu \rho \sigma} R^{\mu \nu \nu}{ }_{k \lambda}=96 \pi^{2} \tau,
\end{aligned}
$$

where $\chi$ is the Euler characteristic and $\tau$ the Hirzebruch signature. Not all these quantities are independent; in four dimensions one has the identity

$$
R_{\mu \nu \lambda \sigma} R^{\mu \nu \lambda \sigma}=C_{\mu \nu \lambda \sigma} C^{\mu \nu \lambda \sigma}+2 R_{\mu \nu} R^{\mu \nu}-\frac{1}{3} R^{2}
$$

Furthermore the expression for the Euler characteristic can be rewritten as

$$
\chi=\frac{1}{32 \pi^{2}} \int \mathrm{d}^{4} x \sqrt{\mathrm{g}}\left[R_{\mu \nu \lambda \sigma} R^{\mu \nu \lambda \sigma}-4 R_{\mu \nu} R^{\mu \nu}+R^{2}\right]
$$

and thus only two curvature-squared terms for the action are independent in four dimensions.

To get more of a feeling for the difference between different higher derivative terms consider the following example. A particular case of riemannian manifold is one of dimension $d$ which is locally isometrically embedded in $(d+1)$-dimensional euclidean space with the canonical euclidean metric. If the manifold is locally convex, the principal curvatures $k_{\lambda}$ are of the same sign everywhere. Then the manifold is the called a locally convex hypersurface [18]. Every point of the manifold admits a neighborhood in which the vectors tangent to the lines of curvature form an orthonormal frame such that

$$
R_{\mu \nu \rho \sigma}=k_{\mu} k_{\nu}\left(\delta_{\nu \sigma} \delta_{\mu \rho}-\delta_{\nu \rho} \delta_{\mu \sigma}\right)
$$


Define $K_{\mu \nu}=k_{\mu} k_{\nu}$. Working out the algebra in four dimensions one obtains

$$
\begin{aligned}
R & =\sum k^{2}-\left(\sum k\right)^{2}, \\
R^{2}= & \left(\sum k^{2}\right)^{2}-2\left(\sum k^{2}\right)\left(\sum k\right)^{2}+\left(\sum k\right)^{4}, \\
R_{\mu \nu}= & \delta_{\mu \nu}\left[k_{\mu}^{2}-\left(\sum k\right) k_{\mu}\right], \\
R_{\mu \nu} R^{\mu \nu}= & \sum k^{4}-2\left(\sum k\right) \sum k^{3}+\left(\sum k\right)^{2} \sum k^{2}, \\
R_{\mu \nu \lambda \sigma} R^{\mu \nu \lambda \sigma}= & 2\left(\sum k^{2}\right)^{2}-2 \sum k^{4}, \\
C_{\mu \nu \lambda \sigma} C^{\mu \nu \lambda \sigma}= & 2\left(\sum k^{2}\right)^{2}-2 \sum k^{4}-2 \sum k^{4}+4\left(\sum k\right) \sum k^{3}-2\left(\sum k\right)^{2} \sum k^{2} \\
& +\frac{1}{3}\left(\sum k^{2}\right)^{2}-2\left(\sum k^{2}\right)\left(\sum k\right)^{2}+\left(\sum k\right)^{4}
\end{aligned}
$$

and therefore

$$
\begin{gathered}
R=2\left(K_{12}+K_{13}+K_{14}+K_{23}+K_{24}+K_{34}\right), \\
R^{2}=4\left(K_{12}+K_{13}+K_{14}+K_{23}+K_{24}+K_{34}\right)^{2}, \\
R_{\mu \nu \lambda \sigma} R^{\mu \nu \lambda \sigma}=2\left(K_{12}^{2}+K_{13}^{2}+K_{14}^{2}+K_{23}^{2}+k_{24}^{2}+K_{34}^{2}\right) \\
R_{\mu \nu \lambda \sigma} R^{\mu \nu \lambda \sigma}-\frac{1}{6} R^{2}=\frac{2}{3}\left[\left(K_{12}-K_{13}\right)^{2}+\left(K_{12}-K_{14}\right)^{2}+\left(K_{12}-K_{23}\right)^{2}+\cdots\right]
\end{gathered}
$$

and

$$
C_{\mu \nu \lambda \sigma} C^{\mu \nu \lambda \sigma}=\frac{2}{3} \sum_{\substack{p \operatorname{airs}(\mu \nu)(\rho \sigma) \\ \mu \neq \nu, \rho \neq \sigma,(\mu \nu) \neq(\rho \sigma)}} \varepsilon(\mu, \nu, \rho, \sigma)\left(K_{\mu \bar{\nu}} K_{\rho \sigma}\right)^{2},
$$

where $\varepsilon(\mu, \nu, \rho, \sigma)$ is equal to 1 if the pairs $(\mu \nu)$ and $(\rho \sigma)$ share one index, and -2 if they do not. This simple example shows some of the difference between $R^{2}, R_{\mu \nu \rho \sigma}^{2}$ and $C_{\mu \nu \rho \sigma}^{2}$. While $R_{\mu \nu \rho \sigma}^{2}$ tries to make all $K_{\mu \nu}$ 's small when inserted in the functional integral, $R_{\mu \nu \lambda_{\sigma}} R^{\mu \nu \lambda \sigma}-\frac{1}{6} R^{2}$ tries to make all the $K_{\mu \nu}$ 's equal to each other, but does not require them to be small. We expect a similar effect from $C_{\mu \nu \lambda_{\sigma}} C^{\mu \nu \lambda \sigma}$. The reasoning here is as follows. Firstly $C_{\mu \nu \lambda \sigma} C^{\mu \nu \lambda \sigma}$ is positive, and so we expect it to be represented by a sum of squares. Secondly it must vanish for spaces which are conformally flat, even though the components of the curvature themselves do not vanish. Thus we expect $C_{\mu \nu \lambda \sigma} C^{\mu \nu \lambda \sigma}$ to be represented by a sum of squares of differences between contributions to the curvature, and its effect in the functional integral will therefore be to make these contributions equal to each other. 
Before discussing the construction of higher derivative terms, we recall the lattice analogue of the Einstein-Hilbert action [12]

$$
I_{\mathrm{R}}=2 \sum_{\text {hinges h }} A_{\mathrm{h}} \delta_{\mathrm{h}},
$$

where $A_{\mathrm{h}}$ is the volume of the hinge and $\delta_{\mathrm{h}}$ is the deficit angle there. The action is the equivalent for a simplicial decomposition of the continuum expression

$$
I_{\mathrm{E}}=\int \mathrm{d}^{4} x \sqrt{g} R .
$$

The deficit angle $\delta_{\mathrm{b}}$ is defined by

$$
\delta_{\mathrm{h}}=2 \pi-\sum_{\substack{\text { 4-simplices } \\ \text { meeting on } \mathrm{h}}} \theta_{\mathrm{d}},
$$

where $\theta_{d}$ is the dihedral angle at the hinge $h$. (In four-dimensional piecewise linear space, the curvature is concentrated on two-dimensional hinges, the triangles.) Let us mention in passing that an alternative lattice action, which also reduces to the Einstein-Hilbert action in the continuum limit, was suggested in ref. [25].

The lattice form of a cosmological constant term, which in the continuum theory reads

$$
\lambda \int \mathrm{d}^{4} x \sqrt{g}
$$

is

$$
\lambda \sum_{\text {d-simplices }} V_{\text {simplex }}=\lambda \sum_{\text {hinges h }} V_{\mathrm{h}},
$$

where $V_{\text {simplex }}$ is the volume of a four-simplex and $V_{\mathrm{h}}$ is the volume associated with each hinge. (This may be defined in a variety of ways, for example using the dual lattice or using a baricentric subdivision. For a full discussion see refs. [16,17]. The only requirement is for a well defined and natural method of dividing the volume of a four-simplex into parts associated with each hinge. Then $V_{\mathrm{h}}$ is the sum of the contributions from the simplices meeting on the hinge $h$. In the continuum limit, when it exists, the particular choice for $V_{h}$ should become irrelevant.)

As discussed in refs. [16], the simplest higher derivative term which can be written down on a simplicial lattice is given by

$$
\sum_{\mathrm{h}} \frac{A_{\mathrm{h}}^{2} \delta_{\mathrm{h}}^{2}}{V_{\mathrm{h}}}
$$

It was shown that this type of term converges to the continuum expression

$$
\frac{1}{4} \int \mathrm{d}^{4} x \sqrt{g} R^{2}
$$

for regular tessellations of the $n$-sphere. However the regular tessellations of $S^{n}$ are 
not able to distinguish between some of the higher derivative terms since one has in this case

$$
R_{\mu \nu \rho \sigma} R^{\mu \nu \rho \sigma} \propto R_{\mu \nu} R^{\mu \nu} \propto R^{2} \propto \frac{1}{r^{2}}
$$

for a sphere of radius $r$. Since the above lattice higher derivative term vanishes if and only if all the deficit angles vanish, that is if and only if space-time is flat, it is in fact really a candidate for [19]

$$
\frac{1}{4} \int \mathrm{d}^{4} x \sqrt{g} R_{\mu \nu \rho \sigma} R^{\mu \nu \rho \sigma} .
$$

A study of parallel transports around a hinge [16] leads to an expression for the Riemann tensor

$$
R_{\mu \nu \rho \sigma}^{(\mathrm{h})}=\frac{A_{\mathrm{h}} \delta_{\mathrm{h}}}{V_{\mathrm{h}}} U_{\mu \nu}^{(\mathrm{h})} U_{\rho \sigma}^{(\mathrm{h})}
$$

where $U_{\mu v}^{(h)}$ is a bivector orthogonal to the hinge h, defined by

$$
U_{\mu \nu}^{(\mathrm{b})}=\frac{1}{2 A_{\mathrm{h}}} \varepsilon_{\mu \nu \rho \sigma} l_{(a)}^{\rho} l_{(b)}^{\rho}
$$

and $l_{(a)}^{\rho}$ and $l_{(b)}^{\rho}$ are the vectors forming two sides of the hinge $h$. This formula for $\boldsymbol{R}_{\mu \nu \rho \sigma}$ is closely related to the one given by Regge [12]. While it does have all the correct symmetries, it does not give full information about the Riemann tensor as it is deduced from a formula which gives only the projection of $R_{\mu \nu \rho \sigma}$ in the plane of the elementary parallel transport loop orthogonal to the hinge $h$. However the value of $R$ obtained by contracting

$$
R^{(\mathrm{h})}=2 \frac{A_{\mathrm{h}} \delta_{\mathrm{h}}}{V_{\mathrm{h}}}
$$

is consistent with the expression due to Regge for the Einstein action.

The use of formula (2.18) for the Riemann tensor on a given hinge gives higher derivative terms which are all proportional to each other

$$
\frac{1}{4} R^{(\mathrm{h})}{ }_{\mu \nu \rho \sigma} R^{(\mathrm{h})^{\mu \nu \rho \sigma}}=\frac{1}{2} R^{(\mathrm{h})}{ }_{\mu \nu} R^{(\mathrm{h})^{\mu \nu}}=\frac{1}{4} R^{(\mathrm{h})^{2}}=\left(\frac{A_{\mathrm{h}} \delta_{\mathrm{h}}}{V_{\mathrm{h}}}\right)^{2} .
$$

Furthermore if one uses the above expression for the Riemann tensor to evaluate the contribution to the Euler characteristic on each hinge one obtains zero, and it is therefore clear that one needs cross-terms involving contributions from different hinges. Even then it seems unlikely that one would obtain the correct integer value for a particular simplicial decomposition by this method. 
Thus we are faced with the puzzling situation that only one higher derivative term of the type considered here can be constructed at a given hinge, while in the continuum there appear to be two independent terms. The next step is then to construct the full Riemann tensor by considering more than one hinge. The simplest possibilities would be to consider all the hinges of a particular simplex or, alternatively, all the hinges that have one point in common. Note that since one uses a coordinate dependent object like (2.19), one should consider contributions from those hinges which can be covered by the same coordinate system. Define the Riemann tensor for a simplex $\mathrm{s}$ as a weighted sum of hinge contributions

$$
\left[R_{\mu \nu \rho \sigma}\right]_{\mathrm{s}}=\sum_{\mathrm{h} \subset \mathrm{s}} \omega_{\mathrm{s}, \mathrm{h}}\left[\frac{A \delta}{V} U_{\mu \nu} U_{\rho \sigma}\right]_{\mathrm{h}},
$$

where the $\omega_{\mathrm{s}, \mathrm{h}}$ are dimensionless weights, to be determined later. After squaring one obtains

$$
\left[R_{\mu \nu \rho \sigma} R^{\mu \nu \rho \sigma}\right]_{\mathrm{s}}=\sum_{\mathrm{h}, \mathrm{h}^{\prime} \subset \mathrm{s}} \omega_{\mathrm{s}, \mathrm{h}^{\omega}} \omega_{\mathrm{s}, \mathrm{h}^{\prime}}\left[\frac{A \delta}{V} U_{\mu \nu} U_{\rho \mathrm{\sigma}}\right]_{\mathrm{h}}\left[\frac{A \delta}{V} U^{\mu \nu} U^{\rho \sigma}\right]_{\mathrm{h}^{\prime}}
$$

Consider two hinges labeled by $i$ and $j$. By using formula (2.19) for the bivectors $U_{\mu \nu}$ the product of the last two square brackets can be worked out

$$
\begin{aligned}
R_{\mu \nu \rho \sigma}^{(i)} R^{(j)^{\mu \nu \rho \sigma}} & \equiv\left[\frac{A \delta}{V} U_{\mu \nu} U_{\rho \sigma}\right]_{(i)}\left[\frac{A \delta}{V} U^{\mu \nu} U^{\rho \sigma}\right]_{(j)} \\
& =\frac{\delta_{i} A_{i} \delta_{j} A_{j}}{V_{i} V_{j}} \frac{1}{4 A_{i}^{2} A_{j}^{2}}[(a \cdot c)(b \cdot d)-(a \cdot d)(b \cdot c)]^{2},
\end{aligned}
$$

where $a$ and $b$ are two edges in hinge $i$, and $c$ and $d$ are two edges in hinge $j$. For the square of the Ricci tensor one needs the expressions

$$
\begin{aligned}
R_{\mu \nu}^{(i)} R^{(j)^{\mu \nu}} \equiv & {\left[\frac{A \delta}{V} U_{\mu}^{\rho} U_{\rho \nu}\right]_{(i)}\left[\frac{A \delta}{V} U^{\sigma \mu} U_{\sigma}^{\nu}\right]_{(j)} } \\
= & \frac{\delta_{i} A_{i} \delta_{j} A_{j}}{V_{i} V_{j}} \frac{1}{16 A_{i}^{2} A_{j}^{2}} \\
\times & {\left[a^{2} c^{2}(b \cdot d)^{2}+a^{2} d^{2}(b \cdot c)^{2}+b^{2} c^{2}(a \cdot d)^{2}+b^{2} d^{2}(a \cdot c)^{2}\right.} \\
& -2\left[a^{2}(b \cdot c)(c \cdot d)(d \cdot b)+b^{2}(a \cdot d)(c \cdot d)(d \cdot a)\right. \\
& \left.+c^{2}(a \cdot b)(b \cdot d)(d \cdot a)+d^{2}(a \cdot b)(b \cdot c)(c \cdot a)\right] \\
& +2[(a \cdot b)(c \cdot d)[(a \cdot c)(b \cdot d)+(a \cdot d)(b \cdot c)]]]
\end{aligned}
$$


for the scalar curvature squared

$$
\begin{aligned}
R^{(i)} R^{(j)} & \equiv\left[\frac{A \delta}{V} U^{\mu \nu} U_{\mu \nu}\right]_{(i)}\left[\frac{A \delta}{V} U^{\rho \sigma} U_{\rho \sigma}\right]_{(j)} \\
& =4 \frac{\delta_{i} A_{i} \delta_{j} A_{j}}{V_{i} V_{j}}
\end{aligned}
$$

for the Euler density

$$
\begin{aligned}
R_{\mu \nu \rho \sigma}^{(i)} R^{(j)^{\alpha \lambda \omega \tau}} \varepsilon^{\mu \nu \kappa \lambda} \varepsilon^{\rho \sigma \omega \tau} & \equiv\left[\frac{A \delta}{V} U_{\mu \nu} U_{\rho \sigma}\right]_{(i)}\left[\frac{A \delta}{V} U_{\kappa \lambda} U_{\omega \tau}\right]_{(j)} \varepsilon^{\mu \nu \kappa \lambda} \varepsilon^{\rho \sigma \omega \tau} \\
& =\frac{\delta_{i} A_{i} \delta_{j} A_{j}}{V_{i} V_{j}} \frac{1}{A_{i}^{2} A_{j}^{2}}\left[\varepsilon_{\mu \nu \rho \sigma} a^{\mu} b^{\nu} c^{\rho} d^{\sigma}\right]^{2}
\end{aligned}
$$

and finally for the Hirzebruch density

$$
\begin{aligned}
R_{\mu \nu \rho \sigma}^{(i)} R^{(j)^{\mu \nu}}{ }_{\kappa \lambda} \varepsilon^{\rho \sigma \kappa \lambda} & \equiv\left[\frac{A \delta}{V} U_{\mu \nu} U_{\rho \sigma}\right]_{(i)}\left[\frac{A \delta}{V} U^{\mu \nu} U_{\kappa \lambda}\right]_{(j)} \varepsilon^{\rho \sigma \kappa \lambda} \\
& =\frac{\delta_{i} A_{i} \delta_{j} A_{j}}{V_{i} V_{j}} \frac{1}{2 A_{i}^{2} A_{j}^{2}} \varepsilon_{\mu \nu \rho \sigma} a^{\mu} b^{\nu} c^{\rho} d^{\sigma}[(a \cdot c)(b \cdot d)-(a \cdot d)(b \cdot c)] .
\end{aligned}
$$

In the above formulae $A_{i}$ and $A_{j}$ are the areas of the triangles $i$ and $j$, respectively.

The question of the weights $\omega_{\mathrm{s}, \mathrm{h}}$ introduced in eq. (2.22) will now be addressed. Consider the expression for the scalar curvature of a simplex defined as

$$
[R]_{\mathrm{s}}=\sum_{\mathrm{h} \in \mathrm{s}} \omega_{\mathrm{s}, \mathrm{h}}\left[2 \frac{A \delta}{V}\right]_{\mathrm{h}}
$$

The natural volume associated with such lattice curvature invariants is the sum of the volumes associated with the hinges in the simplex

$$
V_{\mathrm{s}}=\sum_{\mathrm{h} \subset \mathrm{s}} V_{\mathrm{h}}
$$

Summing the scalar curvature over all simplices, one should recover Regge's expression

$$
\sum_{\mathrm{s}} V_{\mathrm{s}}[R]_{\mathrm{s}}=\sum_{\mathrm{s}} \sum_{\mathrm{h} \subset \mathrm{s}} \omega_{\mathrm{s}, \mathrm{h}}\left[2 \frac{\delta A}{V}\right]_{\mathrm{h}}=2 \sum_{\mathrm{h}} A_{\mathrm{h}} \delta_{\mathrm{h}},
$$


which implies

$$
\sum_{\mathrm{s} \supset \mathrm{h}} \omega_{\mathrm{s}, \mathrm{h}} V_{\mathrm{s}} \frac{A_{\mathrm{h}} \delta_{\mathrm{h}}}{V_{\mathrm{h}}}=A_{\mathrm{h}} \delta_{\mathrm{h}}
$$

Therefore a natural choice for the weights is

$$
\omega_{\mathrm{s}, \mathrm{h}}=\frac{V_{\mathrm{h}}}{N_{\mathrm{s} / \mathrm{h}} V_{\mathrm{s}}}=\frac{V_{\mathrm{h}}}{N_{\mathrm{s} / \mathrm{h}} \Sigma_{\mathrm{h} \subset \mathrm{s}} V_{\mathrm{h}^{\prime}}} .
$$

(One can check this formula for a regular tessellation; there one needs the identity $N_{\mathrm{s} / \mathrm{h}} N_{\mathrm{h}}=N_{\mathrm{h} / \mathrm{s}} N_{\mathrm{s}}$ where $N_{\mathrm{h}}, N_{\mathrm{s}}$ and $N_{\mathrm{h} / \mathrm{s}}$ are the number of hinges, simplices and hinges per simplex, respectively.)

We see that the weighting factors that reproduce Regge's formula for the Einstein action are just the volume fractions occupied by the various hinges in a simplex, which is not surprising. Of course the above formulae are not unique, since one might have done the above construction of higher derivative terms by considering a vertex $p$ instead of a 4-simplex s. The Riemann tensor is then constructed by averaging with the appropriate weights the contributions of different hinges meeting at one point, and the volume $V_{\mathrm{s}}$ becomes the sum of all hinge volume contributions coming from the hinges touching the point.

The above formulae for higher derivative terms are still rather involved. Of course in dealing with the quantum theory one could consider the two simpler expressions which contain some of the structure of the previous terms

$$
\int \sqrt{g} R_{\mu \nu \lambda \sigma} R^{\mu \nu \lambda \sigma} \sim 4 \sum_{\mathrm{h}} V_{\mathrm{h}}\left(\left[\frac{A \delta}{V}\right]_{\mathrm{h}}\right)^{2},
$$

which vanishes if and only if the Riemann tensor projected on all the hinges vanishes (it is in fact a rewriting of the expression (2.14) for the higher derivative term constructed before), and

$$
\int \sqrt{g} C_{\mu \nu \lambda \sigma} C^{\mu \nu \lambda \sigma} \sim \frac{2}{3} \sum_{s} V_{\mathrm{s}} \sum_{\mathrm{h}, \mathrm{h}^{\prime} \subset \mathrm{s}} \varepsilon_{\mathrm{h}, \mathrm{h}^{\prime}}\left(\omega_{\mathrm{s}, \mathrm{h}}\left[\frac{A \delta}{V}\right]_{\mathrm{h}}-\omega_{\mathrm{s}, \mathrm{h}^{\prime}}\left[\frac{A \delta}{V}\right]_{\mathrm{h}^{\prime}}\right)^{2},
$$

which introduces a short-range coupling between deficit angles. The numerical factor $\varepsilon_{h, h^{\prime}}$ is equal to 1 if the two hinges $h, h^{\prime}$ have one edge in common and -2 if they do not. Note that this interaction term has the remarkable property that it requires neighboring deficit angles to have similar values, but it does not require them to be small.

Alternatively, of course the summation can be done over the points $p$, instead of the simplices $\mathrm{s}$, (as in the exact expression of ref. [20] for the Euler characteristic)

$$
\int \sqrt{g} C_{\mu \nu \lambda \sigma} C^{\mu \nu \lambda \sigma} \sim \frac{2}{3} \sum_{p} V_{p} \sum_{\mathrm{h}, \mathrm{h} \supset p} \varepsilon_{\mathrm{h}, \mathrm{K}}\left(\omega_{p, \mathrm{~h}}\left[\frac{A \delta}{V}\right]_{\mathrm{h}}-\omega_{\mathrm{p}, \mathrm{h}}\left[\frac{A \delta}{V}\right]_{\mathrm{h}^{\prime}}\right)^{2} .
$$


Here $V_{p}$ is the volume element associated with the point $p$, and the weighting factors $\omega_{p, h}$ measure the fraction of $V_{p}$ occupied by the hinge $\mathrm{h}$, and are thus equal to $V_{\mathrm{h}} / N_{p / \mathrm{h}} V_{p}$, where $N_{p / \mathrm{h}}$ is the number of points per hinge.

This second choice for the definition of $C^{2}$ (sum over sites) appears in fact more natural when one considers the coupling of gravity to matter fields, which will be represented here for simplicity by a scalar field $\phi(x)$. In the continuum an invariant action, up to terms quadratic in $\phi$, is [4]

$$
I_{\text {matter }}=\int \mathrm{d}^{4} x \sqrt{g}\left[\frac{1}{2} g^{\mu \nu} \partial_{\mu} \phi \partial_{\nu} \phi+\frac{1}{2} m^{2} \phi^{2}+g_{1} R \phi^{2}+g_{2} R^{\mu \nu} \partial_{\mu} \phi \partial_{\nu} \phi+\cdots\right] .
$$

On the skeleton define the fields $\phi_{p}$ living on the sites. If $R$ is defined on the sites, then the third interaction term (proportional to $g_{1}$ ) is just a point coupling term. As far as the first term is concerned, introduce lattice (forward) derivatives

$$
\Delta^{i} \phi_{p}=\left(\phi_{p+i}-\phi_{p}\right) / l_{p, p+i},
$$

where $i$ labels the possible directions in which one can move from a point in a given simplex (there are $d$ of them in $d$ dimensions), and $l_{p, p+i}$ is the length of the edge connecting the two points. The metric $g_{i j}$ at point $p$ in a simplex is

$$
g_{i j}(p)=\frac{1}{2}\left(l_{p, p+i}^{2}+l_{p, p+j}^{2}-l_{p+i, p+j}^{2}\right)
$$

and $\sqrt{g}$ is proportional to the volume of the simplex. The lattice analogue of the first term in the action $I_{\text {matter }}$ of eq. (2.37) is then, in four dimensions,

$$
\sum_{p} \sum_{\mathrm{s} \supset p} V_{\mathrm{s}} g_{i j}(p, \mathrm{~s}) \Delta^{i} \phi_{p} \Delta^{j} \phi_{p}
$$

and double-counting can be avoided by summing only over simplices with sides pointing in the positive lattice direction

In two dimensions the corresponding expression is

$$
\sum_{p} \sum_{\mathrm{t} \supset p} V_{\mathrm{h}} g_{i j}(p, \mathrm{t}) \Delta^{i} \phi_{p} \Delta^{j} \phi_{p}
$$

where $t$ labels the triangles. On a regular triangular lattice as the one in fig. 1 one can associate with each point $p$ two adjacent triangles in the positive $(1,2)$ direction, which can be labeled by $\gamma=1,2$. Then the action is simply

$$
\sum_{p} \sum_{\gamma=1}^{2} V_{p}^{\gamma}\left[\left(\Delta^{1} \phi_{p}\right)^{2}+\left(\Delta^{2} \phi_{p}\right)^{2}+2 \cos \alpha_{12, p}^{\gamma} \Delta^{1} \phi_{p} \Delta^{2} \phi_{p}\right],
$$

where $\alpha_{12, p}^{\gamma}$ is the (dihedral) angle between the two edges $l_{p}^{1}$ and $l_{p}^{2}$ coming out of the point $n$ in the triangle labeled by $\gamma$. 


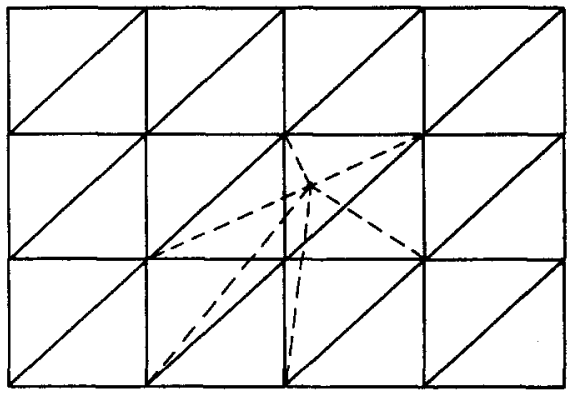

Fig. 1.

\section{The weak field expansion for the tessellation $\alpha_{5}$}

Before discussing numerical calculations in higher derivative lattice gravity, we shall describe some analytic work. This involves the analysis of small fluctuations about a classical background solution. We compute the second variation of the action, which is related to the inverse of the free propagator on the chosen background geometry. The determinant of the matrix representing the second variation of the action gives the contribution from the integration over quadratic fluctuations about the extremum of the action corresponding to the classical solution.

For small fluctuations around flat space, tessellated by a lattice of hypercubes divided into 4-simplices, it has been shown [21] that the lattice propagator, obtained from the second variation of the Regge action, agrees exactly with the continuum result in the weak field (small momentum) limit.

The classical background which we consider here is the surface of a four-sphere tessellated by $\alpha_{5}$, the surface of a five-simplex. The Regge-Einstein action is generalized to include both a cosmological constant and a higher derivative term, the lattice version of $R_{\mu \nu \rho \sigma}^{2}$

$$
I_{\mathrm{L}}=\sum_{\text {hinges h }}\left[\lambda V_{\mathrm{h}}-2 k \delta_{\mathrm{h}} A_{\mathrm{h}}+4 b \frac{A_{\mathrm{h}}^{2} \delta_{\mathrm{h}}^{2}}{V_{\mathrm{h}}}\right] .
$$

This lattice action is the analogue of the continuum action

$$
I=\int \mathrm{d}^{4} x \sqrt{g}\left[\lambda-k R+b R_{\mu \nu \rho \sigma} R^{\mu \nu \rho \sigma}\right] .
$$

We have chosen not to include a term representing the square of the Weyl tensor, since it is more difficult to treat because it couples neighbouring hinges, and our tessellation is unrealistically small for such a term. 


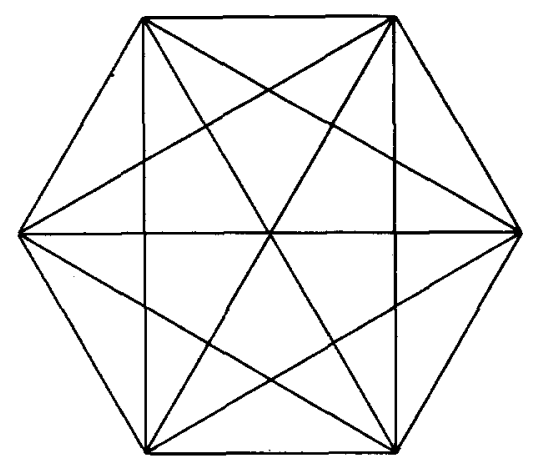

Fig. 2.

The simplicial complex $\alpha_{5}$ has 6 vertices all linked to each other, giving a total of 15 edge lengths. It contains 20 triangular hinges, 15 tetrahedra and 5 4-simplices, and is shown in fig. 2. To find a classical solution, we assume that all edges are of length $l$. Evaluation of the lattice action then gives

$$
I_{\mathrm{L}}=\frac{1}{16} \sqrt{5} \lambda l^{4}-10 \sqrt{3} k \delta l^{2}+960 \sqrt{5} b \delta^{2}
$$

where $\delta=2 \pi-3 \cos ^{-1}\left(\frac{1}{4}\right)$ is the deficit angle at each triangle. The classical solution is obtained by solving

$$
\frac{\partial I_{\mathrm{L}}}{\partial l^{2}}=0
$$

and gives $l^{2}=l_{0}^{2} \equiv 16 \theta k / \lambda$, where $\theta=\sqrt{15} \delta$. (Note that a classical solution with no higher derivative term is also a solution in the presence of the higher derivative term, as in the continuum [1].)

We then allow for small variations about the classical background solution by setting

$$
l_{i}=l_{0}\left(1+\varepsilon_{i}\right), \quad i=1,2, \ldots, 15,
$$

with $\varepsilon_{i} \ll 1$, and evaluate the lattice action up to second order in $\varepsilon$. The various terms in the lattice action are given by the following expressions. The total volume is

$$
\sum_{\mathrm{h}} V_{\mathrm{h}}=\frac{1}{16} \sqrt{5} l_{0}^{4}\left[1+\frac{4}{15} \sum_{i} \varepsilon_{i}-\frac{18}{25} \sum_{i} \varepsilon_{i}^{2}+\frac{8}{25} \sum_{\substack{i \neq j \\ i, j \text { neighboring }}} \varepsilon_{i} \varepsilon_{j}-\frac{4}{75} \sum_{\substack{i j \\ i, j \text { opposite }}} \varepsilon_{i} \varepsilon_{j}\right] \text {. }
$$


The Einstein action depends on

$$
\begin{aligned}
\sum_{\mathrm{h}} A_{\mathrm{b}} \delta_{\mathrm{h}}=-\sqrt{\frac{1}{5}} \theta I_{0}^{2} & {\left[-5-\frac{2}{3} \sum_{i} \varepsilon_{i}+\left(\frac{1}{\theta}+\frac{5}{9}\right) \sum_{i} \varepsilon_{i}^{2}\right.} \\
& \left.-\left(\frac{1}{\theta}+\frac{2}{9}\right) \sum_{\substack{i \neq j \\
i, j \text { neighboring }}} \varepsilon_{i} \varepsilon_{j}+\frac{1}{\theta} \sum_{\substack{i j \\
i, j \text { opposite }}} \varepsilon_{i} \varepsilon_{j}\right]
\end{aligned}
$$

and the higher derivative term is proportional to

$$
\begin{aligned}
\sum_{\mathrm{h}} \frac{A_{\mathrm{h}}^{2} \delta_{\mathrm{h}}^{2}}{V_{\mathrm{h}}}=4 \sqrt{\frac{1}{5}} \theta^{2}[20 & +\left(\frac{2512}{225}-\frac{136}{5 \theta}+\frac{288}{\theta^{2}}\right) \sum_{i} \varepsilon_{i}^{2} \\
& +\left(\frac{904}{225}+\frac{112}{5 \theta}-\frac{126}{\theta^{2}}\right) \sum_{\substack{i \neq j \\
i, j \text { neighboring }}} \varepsilon_{i} \varepsilon_{j} \\
& \left.+\left(\frac{368}{225}-\frac{104}{5 \theta}+\frac{72}{\theta^{2}}\right) \sum_{\substack{i j \\
i, j \text { opposite }}} \varepsilon_{i} \varepsilon_{j}\right] .
\end{aligned}
$$

(The calculation of these quantities was done by hand using the formulae in refs. [16] and [17], and was checked using the algebraic manipulation program Macsyma.) When the value of $l_{0}^{2}$ from the classical solution is inserted, the total action becomes

$$
I_{\mathrm{L}} \approx 16 \sqrt{\frac{1}{5}} \theta^{2}\left[c+a \sum_{i} \varepsilon_{i}^{2}+2 \beta \sum_{\substack{i \neq j \\ i, j \text { neighboring }}} \varepsilon_{i} \varepsilon_{j}+2 \gamma \sum_{\substack{i j \\ i, j \text { opposite }}} \varepsilon_{i} \varepsilon_{j}\right],
$$

with

$$
\begin{aligned}
c & =5\left(-\frac{k^{2}}{\lambda}+4 b\right), \\
\alpha & =-\frac{k^{2}}{\lambda}\left(\frac{112}{45}-\frac{2}{\theta}\right)+8 b\left(\frac{314}{225}-\frac{17}{5 \theta}+\frac{36}{\theta^{2}}\right), \\
2 \beta & =-\frac{k^{2}}{\lambda}\left(-\frac{52}{45}+\frac{2}{\theta}\right)+8 b\left(\frac{113}{225}+\frac{14}{5 \theta}-\frac{63}{4 \theta^{2}}\right), \\
2 \gamma & =-\frac{k^{2}}{\lambda}\left(\frac{4}{15}-\frac{2}{\theta}\right)+8 b\left(\frac{46}{225}-\frac{13}{5 \theta}+\frac{9}{\theta^{2}}\right) .
\end{aligned}
$$


Thus we may write the second variation of the action as

$$
16 \sqrt{\frac{1}{3}} \theta^{2} \varepsilon_{i} M_{i j} \varepsilon_{j}
$$

with $M_{i j}$ a $15 \times 15$ symmetric matrix with $\alpha$ on the diagonal and $\beta$ or $\gamma$ in the off-diagonal position, depending on whether $i$ and $j$ represent neighboring or non-adjacent edges in $\alpha_{5}$. The eigenvalues of $M$ are given by

$$
\begin{array}{ll}
\lambda_{0}=\alpha-2 \beta+\gamma & \text { (multiplicity 9) } \\
\lambda_{1}=\alpha+2 \beta-3 \gamma & \text { (multiplicity 5) } \\
\lambda_{2}=\alpha+8 \beta+6 \gamma & \text { (multiplicity 1). }
\end{array}
$$

As discussed by Hartle [22], the high degree of degeneracy of the eigenvalues reflects the invariance of the lattice under the symmetry group of the five-simplex. The multiplicities correspond to the dimensions of the irreducible components of the permutation representations of the symmetry group of the vertices of $\alpha_{5}$. Inserting the actual value for the deficit angle $\delta$ one obtains the final answer for the eigenvalues

$$
\begin{aligned}
& \lambda_{0}=-3.2234 \frac{k^{2}}{\lambda}+6.8437 b, \\
& \lambda_{1}=-1.2659 \frac{k^{2}}{\lambda}+16.3197 b, \\
& \lambda_{2}=+1.3333 \frac{k^{2}}{\lambda}+32.1422 b .
\end{aligned}
$$

For $b=0$ (no higher derivative term) 14 out of 15 eigenvalues are negative. This indicates a strong instability: the dominant edge length configurations are presumably far away from the symmetric and smooth one. Most of the lattice degrees of freedom go into reproducing the unbounded conformal mode, and not enough positive eigenvalues are left to represent the physical degrees of freedom. For $b \geqslant 0.0776 k^{2} / \lambda$ the eigenvalue $\lambda_{1}$ becomes positive, and for $b \geqslant 0.4710 k^{2} / \lambda$ all eigenvalues are positive and the starting configuration of edges is stable. This would not have been the case if the theory had a tachyon or some other instability. Thus even for a finite number of degrees of freedom the theory has what one might call a phase transition, corresponding to the hessian matrix developing a zero eigenvalue for small enough $b$. It could be that the interesting region in a more realistic model (i.e. with more degrees of freedom) is close to this "transition" point. It would be interesting to see how the density of eigenvalues behaves as the tessellation is made 
finer by the inclusion of more points. In conclusion we mention that Hartle [22] has studied the behavior of the Regge-Einstein action with a cosmological constant term on $\alpha_{5}$ (and other tessellations in four dimensions) without relying on the expansion around the equilateral configuration of edges. He finds that in all cases the stationary point of the Regge action with a cosmological term corresponding to all edges of equal length is a saddle point and not a local minimum.

\section{Quantum gravity beyond perturbation theory}

In principle a natural setting for lattice quantum gravity calculations would be a random lattice, in which the coordination number at each site is itself a random variable. Unfortunately such a lattice is rather difficult to deal with, both analytically and numerically [23].

Another possibility is to use the regular tessellations of the $n$-sphere [24]. Since the maximum number of edges allowed in such tessellations is not very large, a refinement of the same could be achieved by considering further regular subdivisions, such as the barycentric one. Thus the degree of irregularity would be kept at a minimum.

A third possibility is to start with a hypercubical lattice, which can be made topologically equivalent to a hypertorus, by identifying opposite faces. Finite volume effects are minimized for this lattice, since the boundary is formed by a replica of the same lattice. The advantage of the hypercubic lattice lies in the fact that the number of edges can be increased arbitrarily, keeping the local incidence matrix unchanged. If the theory has some reasonable continuum limit, then this limit should not depend on the detailed lattice structure at short distances and on the nature of the boundary conditions. Of course different topologies can be obtained by changing the boundary conditions.

The lattice action for gravity described in the previous sections does not contain terms which allow tunnelling from one spacetime topology to another. Thus initially one would like to keep the topology fixed, and vary the metric within the given sector. Eventually it will be important to verify that the results obtained do not depend on the particular topology chosen (i.e. on the nature of the infrared cutoff) just as they should not depend on the detailed lattice transcription of the continuum action (i.e. on the nature of the ultraviolet cutoff). This is likely to happen for correlations of local operators over distances that are much smaller then the size of the system, and much larger than the average separation between spacetime points. In fact the renormalization properties of the lattice operators can be extracted by looking at the dependence of the low-energy effective hamiltonian (and its correlation functions) on the ultraviolet cutoff. Here by low energy one means energies that are still well above the infrared cutoff set by the finite box (universe) size.

It is not clear at the present moment how the integration over topologies should be performed, and how the weighting should be assigned. For a thorough discussion 


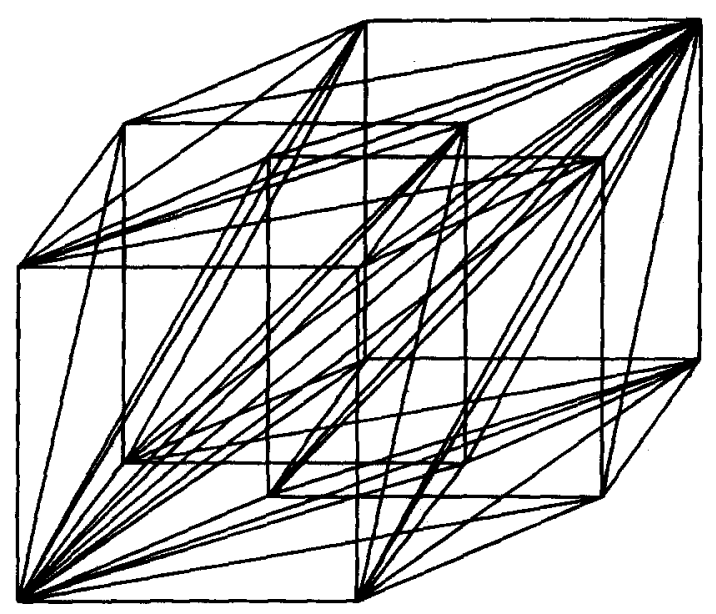

Fig. 3.

of this point see ref. [22]. Arguments have been given for suggesting that a sum over topologies in Regge calculus cannot give a finite functional integral, because the number of manifolds with a given topology increases too rapidly as a function of the number of simplices [25].

The asymptotic freedom of higher derivative gravity further restricts the short distance fluctuations in the metric, implying that the field configurations become smooth at the scale of the ultraviolet cutoff [9]. Furthermore it seems unlikely that a unitary theory can be defined by summing over topologies [25]. The time-slice factorization property of the functional integral needed to construct a time evolution operator no longer holds if this summation is performed, even if the action is reflection positive [26]. This is connected with the fact that the weighting factors for individual topologies, being necessarily topological invariants, are only globally defined.

In the following only results obtained with the hypercubic lattice will be discussed. In fig. 3 a single hypercube is drawn, with the relevant body principals, face diagonals, body diagonals and hyperbody diagonals [21]. The diagonals have to be introduced to make the lattice rigid. Otherwise the values of the edge lengths do not determine the angles, and therefore the geometry, uniquely. The hypercube is then replicated in four directions to construct the full skeleton.

After having chosen an appropriate lattice, the next step is to define and evaluate the functional integral for simplicial quantum gravity restricted to a manifold of fixed topology, say a hypertorus

$$
Z=\int \mathrm{d} \mu[l] \mathrm{e}^{-I[l]}
$$


In the following the scale invariant form of the measure $[17,27]$

$$
\int \mathrm{d} \mu_{\varepsilon}[l]=\prod_{i} \int_{0}^{\infty} \frac{\mathrm{d} l_{i}^{2}}{l_{i}^{2}} F_{\varepsilon}[l]
$$

will be used, where $F_{\mathrm{e}}[l]$ is a rather complicated function of the edge lengths, with the property that it is equal to one when the triangle inequalities and their higher dimensional analogues for the simplicial complex are satisfied, and zero otherwise. These inequalities ensure that the edge lengths, triangle areas, tetrahedron and four-simplex volumes are positive. The positive real parameter $\varepsilon$ is introduced as an ultraviolet cutoff at small edge lengths: the function $F_{e}[l]$ is zero if any of the edges is equal or less than $\varepsilon$.

Of course the measure suggested above is not unique, but is certainly the most attractive one, since it is local and scale invariant as the continuum measure [15], and integrates directly over the elementary lattice degrees of freedom, the conformal factors $\phi_{i}=\ln \left(l_{i} / l_{0}\right)$. Other measures one might consider would involve an integration over edge lengths divided by some volume to the appropriate power, such that the total measure is scale invariant. However there are several volumes that are touching a given edge, and the measure then becomes rather complicated, involving some odd powers of volumes in the denominator.

The continuum limit, in four dimensions is taken by requiring that the local curvature be small on the scale of the local lattice spacing, which is equivalent to imposing

$$
\left|\frac{A_{\mathrm{h}} \delta_{\mathrm{h}}}{V_{\mathrm{h}}}\right| \ll \frac{1}{\sqrt{V_{\mathrm{h}}}}
$$

and implies

$$
\frac{A_{\mathrm{h}}^{2} \delta_{\mathrm{h}}^{2}}{V_{\mathrm{h}}} \ll 1,
$$

a condition which can be achieved by having the coefficient of the action term of equation (2.34) large. If these conditions are not met, the results are likely to depend strongly on the detailed structure of the ultraviolet cutoff (i.e. choice of lattice structure and lattice transcription of the continuum action). Note also that smoothness on the scale of the cutoff does not imply flatness.

A possible approach to evaluate the functional integral is by using numerical Monte Carlo methods, that do not rely on a (possibly non-existent) expansion in a small parameter. Then the edges of the skeleton are varied individually (or in small groups) by a small amount, and the difference in action is compared. If the action is lowered, the new edge value is accepted, if it is raised then the new edge length is only accepted with a probability given by the exponential of the action difference. 
The same procedure is then applied to another edge, and so on. After many edges have been changed, the probability distribution for the edges approaches the equilibrium one, if it exists. For a more detailed discussion of the procedure see ref. [11].

The existence of the functional integral itself does not appear to be a necessary requirement for a consistent quantum theory, since physical observables are obtained from Green functions which in turn are functional derivatives of $-(1 / V) \ln Z$. (One familiar example is non-compact lattice electrodynamics, which leads to finite answers for gauge-invariant quantities, even without gauge-fixing.) An alternative to the path integral quantization method is the stochastic formulation for gravity. Whenever the functional integral (4.1) exists, the corresponding averages can also be obtained by solving the set of stochastic differential equations (Langevin equation) [17]

$$
\frac{1}{l_{\mathrm{p}}(t)} \frac{\mathrm{d} l_{\mathrm{p}}(t)}{\mathrm{d} t}=-l_{\mathrm{p}}(t) \frac{\delta I[l]}{\delta l_{\mathrm{p}}(t)}+\sqrt{2} \eta_{\mathrm{p}}(t) .
$$

Here $t$ is a fictitious fifth time, and the field $\eta_{p}(t)$ is a gaussian white noise with zero mean and unit variance: $\left\langle\eta_{\mathrm{p}}(t)\right\rangle=0,\left\langle\eta_{\mathrm{p}}(t) \eta_{q}\left(t^{\prime}\right)\right\rangle=\delta_{\mathrm{p}, q} \delta\left(t-t^{\prime}\right)$. The constraint that the triangle inequalities be satisfied implies that the force term is infinite whenever they are violated. Averaging over the noise $\eta_{\mathrm{p}}(t)$ reproduces then the averages computed by the functional integral method, in the limit of large times $[28,29]$. For pure $R$-gravity without a cosmological constant term the above equation reduces to [17]

$$
\frac{1}{l_{\mathrm{p}}(t)} \frac{\mathrm{d} l_{\mathrm{p}}(t)}{\mathrm{d} t}=k l_{\mathrm{p}}^{2}(t) \sum_{\mathrm{h} J l_{\mathrm{p}}} \delta_{\mathrm{h}}(t) \cot \theta_{\mathrm{ph}}(t)+\sqrt{2} \eta_{\mathrm{p}}(t),
$$

where the sum is over triangles, labeled by $h$, meeting on the common edge $p$, and $\theta_{\mathrm{ph}}$ is the angle in the hinge $\mathrm{h}$ opposite to the edge $\mathrm{p}$. There is no reason of course for this process to converge, given the unboundedness of the gravitational action both on the lattice and in the continuum.

Let us look at the linearized form of the Langevin equation for higher derivative gravity in the continuum. In the case of the pure Einstein action the equations have been derived independently by the authors of ref. [30]. The relevant action is given in equation (1.7). The corresponding Langevin equation is

$$
\dot{h}_{\mu \nu}(x, t)=-\frac{\delta I[h]}{\delta h_{\mu \nu}}(x, t)+\eta_{\mu \nu}(x, t)
$$

with

$$
\begin{aligned}
\left\langle\eta_{\mu \nu}(x, t)\right\rangle & =0 \\
\left\langle\eta_{\mu \nu}(x, t) \eta_{\rho \sigma}\left(x^{\prime}, t^{\prime}\right)\right\rangle & =2 I_{\mu \nu \rho \sigma} \delta^{(4)}\left(x-x^{\prime}\right) \delta\left(t-t^{\prime}\right),
\end{aligned}
$$


with $I_{\mu \nu \rho \sigma}=\frac{1}{2}\left(\delta_{\mu \nu} \delta_{\rho \sigma}-\delta_{\nu \rho} \delta_{\mu \sigma}\right)$. By separating the spin-2, spin-1 and spin-0 components

$$
h_{\mu \nu}^{(i)}=P_{\mu \nu \alpha \beta}^{(i)} h_{\mu \nu}, \quad i=0,1,2
$$

and going to momentum space one gets

$$
\begin{aligned}
& \dot{h}_{\mu \nu}^{(2)}(q, t)=-\left[\frac{1}{2} k q^{2}+\frac{1}{2} a q^{4}\right] h_{\mu \nu}^{(2)}(q, t)+\eta_{\mu \nu}^{(2)}(q, t), \\
& \dot{h}_{\mu \nu}^{(1)}(q, t)=h_{\mu \nu}^{(1)}(q, t)+\eta_{\mu \nu}^{(1)}(q, t), \\
& \dot{h}_{\mu \nu}^{(0)}(q, t)=-\left[-k q^{2}+2 b q^{4}\right] h_{\mu \nu}^{(0)}(q, t)+\eta_{\mu \nu}^{(0)}(q, t),
\end{aligned}
$$

where $\eta_{\mu \nu}^{(i)}(q, t)$ is the projection of the noise $\eta_{\mu \nu}$ in the spin- $i$ sector. The spin-1 mode feels no force term, and thus performs a random walk with no constraint, and does not couple to the other two modes. The spin-2 mode is also well behaved, while the spin-0 mode blows up exponentially in time if the coupling $b$ is not large enough. Since the Langevin equation is linear, the solution is easy to write down

$$
h_{\mu \nu}(q, t)=\int_{-\infty}^{t} \mathrm{~d} t^{\prime} K_{\mu \nu \rho \sigma}\left(q, t-t^{\prime}\right) \eta_{\mu \nu}\left(q, t^{\prime}\right),
$$

where the kernel $K$ is given by

$$
K_{\mu \nu \rho \sigma}(q, t)=\left[\exp -\left[\frac{1}{2} k q^{2}+\frac{1}{2} a q^{4}\right] t P^{(2)}+P^{(1)}+\exp -\left[-k q^{2}+2 b q^{4}\right] t P^{(0)}\right]_{\mu \nu \rho \sigma} .
$$

The Langevin process thus converges for momenta $q^{2}>k / 2 b$, and the runaway solutions in the spin-0 sector can be restricted to arbitrarily small $q^{2}$, by taking $b$ to be large enough. This is a consequence of the boundedness and renormalizability of the higher derivative gravity action. It remains to be seen what the effect of the neglected higher order terms in $h_{\mu \nu}$ (there is an infinite number of them) is on the convergence. (For the regular tessellation $\alpha_{5}$ discussed above no negative eigenvalues in the quadratic fluctuation matrix are found for $a=4 b$ large enough.)

\section{Numerical results in four dimensions}

In four dimensions the action for pure higher derivative gravity on a simplicial lattice was described in sect. 2. From eqs. (2.34) and (2.35) the full lattice action is

$$
\begin{aligned}
I[l]= & \sum_{\text {hinges h }}\left[\lambda V_{\mathrm{h}}-2 k \delta_{\mathrm{h}} A_{\mathrm{h}}+4 b \frac{A_{\mathrm{h}}^{2} \delta_{\mathrm{h}}^{2}}{V_{\mathrm{h}}}\right] \\
& +\frac{1}{3}(a-4 b) \sum_{\text {points } \mathrm{p}} V_{p} \sum_{\text {hinges h }, \mathrm{h}^{\prime} \supset \mathrm{p}} \varepsilon_{\mathrm{h}, \mathrm{h}}\left[\omega_{\mathrm{h}} \frac{\mathrm{A}_{\mathrm{h}} \delta_{\mathrm{h}}}{\mathrm{V}_{\mathrm{h}}}-\omega_{\mathrm{h}^{\prime}} \frac{\mathrm{A}_{\mathrm{h}^{\mathrm{h}}} \delta_{\mathrm{h}^{\mathrm{h}}}}{\mathrm{V}_{\mathrm{h}^{\prime}}}\right]^{2}
\end{aligned}
$$


and is the lattice analogue of the continuum action

$$
I=\int \mathrm{d}^{4} x \sqrt{g}\left[\lambda-k R+b R_{\mu \nu \rho \sigma} R^{\mu \nu \rho \sigma}+\frac{1}{2}(a-4 b) C_{\mu \nu \rho \sigma} C^{\mu \nu \rho \sigma}\right] .
$$

In the quantum theory one is interested in evaluating averages from the functional integral

$$
Z=\int \mathrm{d} \mu[l] \mathrm{e}^{-I[l]}
$$

with the measure given by eq. (4.2). The lattice is chosen to be regular and built out of rigid hypercubes.

Whenever the functional integral exists, all the edge lengths can be rescaled $l_{i} \rightarrow(k / \lambda)^{1 / 2} l_{i}$ using the scale invariance of the measure, and one gets

$$
Z[\lambda, k, a, b, \varepsilon]=Z\left[\frac{k^{2}}{\lambda}, \frac{k^{2}}{\lambda}, a, b,\left(\frac{\lambda}{k}\right)^{1 / 2} \varepsilon\right] .
$$

If $\varepsilon$ can be sent to zero, then $Z$ can depend only on the dimensionless couplings $k^{2} / \lambda, a$ and $b$, once all lengths are expressed in units of the length scale $l_{0} \equiv$ $(k / \lambda)^{1 / 2}$. (This is certainly true for fixed $k$ and large enough $\lambda, a$ and $b$.) The one-loop perturbative results of ref. [7] then indicate that the theory is asymptotically free in $a$ and $b$. They also suggest that the renormalized, effective low-energy cosmological constant is zero, if the bare one is chosen to be small to start with.

If the functional integral exists for $\varepsilon=0$, then the scale invariance of the measure implies the identity

$$
k\left\langle\sum_{\mathrm{h}} \delta_{\mathrm{h}} A_{\mathrm{h}}\right\rangle=\lambda\left\langle\sum_{\mathrm{h}} V_{\mathrm{h}}\right\rangle
$$

The lattice is chosen of size $N \times N \times N \times N$ with $15 N^{4}$ edges, and up to now only the cases $N=2$ (240 edges) and $N=4$ (3840 edges) have been considered. Periodic boundary conditions are used, and the topology is therefore restricted to a hypertorus. Some of the results discussed here were already presented in ref. [27]. For the formulation of the higher derivative terms baricentric volumes [16] are used, since their expression in terms of the edge lengths is simplest. A time-discretized form of the Langevin evolution equation (4.5)

$$
l_{\mathrm{p}}^{(k+1)}=l_{\mathrm{p}}^{(k)} \exp \left[-\varepsilon \frac{\delta I[l]}{\delta l_{\mathrm{p}}^{(k)}}+\sqrt{2 \varepsilon} \eta_{\mathrm{p}}^{(k)}\right]
$$


was used, where $\varepsilon$ is the step size in time (chosen between $10^{-3}$ and $10^{-4}$ ), and

$$
\begin{aligned}
\left\langle\eta_{\mathrm{p}}^{(k)}\right\rangle & =0, \\
\left\langle\eta_{\mathrm{p}}^{(k)} \eta_{q}^{(l)}\right\rangle & =\delta_{p, q^{\delta^{k, l}}}
\end{aligned}
$$

Also $l_{\mathrm{p}}^{(k+1)}=l_{\mathrm{p}}^{(k)}$ whenever the generalized triangle inequalities are violated.

First consider the case in which all the couplings are zero: $a=b=k=\lambda=0$. Then the total action is zero, and variations in the edge lengths are only constrained by the measure. The edges then perform a constrained random walk, and the situation corresponds to what might be called "random (or disordered) space-time". (To compute the averages, 1000 passes were performed on the $2^{4}$ lattice, discarding the first 500.) While initially the different edges have length $1, \sqrt{2}, \sqrt{3}, 2$ depending on the type (corresponding to a lattice of flat hypercubes), soon they all become roughly of equal average magnitude. (After 1000 passes the four edge types have average value $\sqrt{\left\langle l_{a}^{2}\right\rangle}=1.52,1.50,1.49$ and 1.50 , respectively.) Other quantities of interest are the average curvature $\Re$

$$
\Re=\left\langle l^{2}\right\rangle \frac{\left\langle 2 \Sigma_{\mathrm{h}} \delta_{\mathrm{h}} A_{\mathrm{h}}\right\rangle}{\left\langle\Sigma_{\mathrm{h}} V_{\mathrm{h}}\right\rangle}
$$

and the average curvature squared $\Re^{2}$

$$
R^{2}=\left\langle l^{2}\right\rangle^{2} \frac{\left\langle 4 \sum_{\mathrm{h}} \delta_{\mathrm{h}}^{2} A_{\mathrm{b}}^{2} / V_{\mathrm{h}}\right\rangle}{\left\langle\sum_{\mathrm{h}} V_{\mathrm{h}}\right\rangle},
$$

which are both dimensionless quantities, since they both have been expressed in units of the average edge length. Also of interest is the average deficit angle $\left\langle\delta_{\mathrm{h}}^{2}\right\rangle$ and the average volume of a 4-simplex, in units of the average edge length $\sqrt{\left\langle l^{2}\right\rangle}$. Results for these quantities are displayed in table 1.

Remarkably, at strong coupling the system develops an average negative curvature. Also the value of $\Re^{2}$ is quite large, indicating a significant deviation from flat space behavior. The ratio $\left\langle V_{h}\right\rangle /\left\langle l^{2}\right\rangle^{2}=0.0064$ is also substantially larger than the initial flat space ratio $\left(\frac{1}{50}\right) /\left(\frac{32}{15}\right)^{2}=0.0044$. (The factor of 50 arises because there are 50 hinges per hypercube.)

TABLE 1

Results for the 4-dimensional model with $\lambda=k=a=b=0$

\begin{tabular}{cccc}
\hline $50\left\langle V_{\mathrm{h}}\right\rangle /\left\langle l^{2}\right\rangle^{2}$ & $\Re$ & $\Re^{2}$ & $\left\langle\delta_{\mathrm{h}}^{2}\right\rangle$ \\
\hline $0.32(2)$ & $-10.36(1.20)$ & $58200(600)$ & $3.52(9)$ \\
\hline
\end{tabular}


TABLE 2

Results for the 4-dimensional model with $2 k=1,4 b=0.005, a=4 b$

\begin{tabular}{ccccc}
\hline$\lambda$ & $50\left\langle V_{\mathrm{h}}\right\rangle /\left\langle l^{2}\right\rangle^{2}$ & $\Re$ & $\Re^{2}$ & $\left\langle\delta_{\mathrm{h}}^{2}\right\rangle$ \\
\hline 2.0 & $0.33(2)$ & $-0.612(61)$ & $8900(220)$ & $0.64(12)$ \\
1.5 & $0.33(2)$ & $-0.420(42)$ & $8660(260)$ & $0.62(11)$ \\
1.0 & $0.08(2)$ & $132(22)$ & $134000(13600)$ & $1.65(30)$ \\
0.5 & $0.11(2)$ & $123(14)$ & $143000(11600)$ & $2.45(35)$ \\
\hline
\end{tabular}

A measure of the "roughness" of space-time is given by the dimensionless ratio of curvature over square root of curvature squared

$$
\frac{\mathscr{R}}{\sqrt{\mathscr{R}^{2}}}=\frac{\left\langle 2 \sum_{\mathrm{h}} \delta_{\mathrm{h}} A_{\mathrm{h}}\right\rangle}{\left\langle\sum_{\mathrm{h}} V_{\mathrm{h}}\right\rangle} /\left(\frac{\left\langle 4 \sum_{\mathrm{h}} \delta_{\mathrm{h}}^{2} A_{\mathrm{h}}^{2} / V_{\mathrm{h}}\right\rangle}{\left\langle\sum_{\mathrm{h}} V_{\mathrm{h}}\right\rangle}\right)^{1 / 2},
$$

which can be estimated at about $0.042(8)$.

For intermediate coupling some results are displayed in tables 2 and 3. Table 2 shows the averages for $4 b=0.005$ and table 3 for $4 b=(2 k)^{2} / 4 \lambda$. In all cases $2 k$ is fixed to be $1, \lambda$ is varied, and $a-4 b$ is set equal to zero (pure $R_{\mu \nu \rho \sigma}^{2}$ ). The averages were obtained by doing 2000 passes on the $2^{4}$ lattice, and discarding the first 1000 passes in the final averaging. Results on the $4^{4}$ lattice were then obtained by using the final, duplicated, $2^{4}$ lattice as starting configuration. While these results are comparable within errors with those on the smaller lattice, the low statistics (50 passes) is not sufficient yet to draw any conclusion about finite size effects, even though they appear to be small for the quantities measured.

For small higher derivative coupling ( $4 b=0.005$, table 2$)$ the average curvature $\Re$ could depend very strongly on the value of the bare cosmological constant $\lambda$. The large values for the curvature squared $\Re^{2}$, at least for $\lambda=1.0$ and 0.5 , indicate that, for this choice of coupling constants, the geometry of space-time is not well approximated by a smooth metric. This is in turn an indication that with the Einstein and cosmological constant term only, one is in general far from the lattice continuum limit, as discussed in the first section. It appears also that perhaps the

TABLE 3

Results for the 4-dimensional model with $2 k=1, b=k^{2} / 4 \lambda, a=4 b$

\begin{tabular}{ccccc}
\hline$\lambda$ & $50\left\langle V_{\mathrm{h}}\right\rangle /\left\langle l^{2}\right\rangle^{2}$ & $\Re$ & $\Re^{2}$ & $\left\langle\delta_{\mathrm{h}}^{2}\right\rangle$ \\
\hline 1.5 & $0.24(4)$ & $-0.044(17)$ & $496(48)$ & $0.021(5)$ \\
1.0 & $0.18(4)$ & $-0.031(44)$ & $432(45)$ & $0.013(3)$ \\
0.5 & $0.17(3)$ & $-0.030(48)$ & $208(64)$ & $0.006(2)$ \\
\hline
\end{tabular}


average curvature can be made to vanish by choosing $\lambda$ appropriately, but this would require fine-tuning. In fact the jump in $\Omega$ is so large, that it appears to be indicative of a discontinuous transition. The transition could be connected with the lowest eigenvalue of the quadratic fluctuation matrix becoming zero and then negative, as in the case of the regular tessellation $\alpha_{5}$ discussed previously.

On the other hand, for larger $b$ (table 3 ) the curvature $R$ is quite uniformly small and negative, and appears to still decrease slightly when going from the $2^{4}$ lattice to the $4^{4}$ lattice. $\Re^{2}$ is now substantially smaller, an indication that the field configurations are becoming smoother. As in the two-dimensional case [31] the sum rule (5.5) is not satisfied, due presumably to the absence of classical solutions to the higher derivative continuum field equations with a $\lambda$-term on a 4-torus.

To compute the renormalized, effective low-energy, cosmological constant in units of the Planck mass one needs to determine the renormalized value of Newton's constant. Experimentally it is known that at low energies the dimensionless ratio $\lambda_{R} / k_{R}^{2}$ is about $10^{-120}$ or less. The renormalized cosmological constant $\lambda_{R}$ is obtained from the average curvature $\Re$. The renormalized Newton's constant can be obtained by computing the connected edge two-point function at geodesic distance $d$

$$
G_{\alpha \beta}=\frac{1}{\left\langle l^{2}\right\rangle^{2}}\left\langle l_{\alpha}^{2}(d) l_{\beta}^{2}(0)\right\rangle_{c} \rightarrow T_{\alpha \beta} \frac{k_{\mathrm{R}}^{-1}}{d^{2}} \quad \text { as } d \rightarrow \infty,
$$

where $\alpha$ and $\beta$ label the different edge types at one point on the lattice (body principal, face diagonal, etc.), and $T_{\alpha \beta}$ is the appropriate spin-two projection matrix. (If particles of other spin are contained in the correlation function (5.11), they can be isolated by diagonalizing the propagation matrix $G_{\alpha \beta}$.) Because of the asymptotic freedom of higher derivative gravity theory, the physical dimensionless ratio $\lambda_{\mathrm{R}} / k_{\mathrm{R}}^{2}$ is a computable number, and could turn out to be fairly insensitive to the value of the bare couplings. Given the small lattice that was employed in the present study, it appears difficult to reliably extract a value for $k_{\mathrm{R}}$. Still, the present numerical results on the $4^{4}$ lattice suggest that the curvature expressed in units of $k_{\mathrm{R}}$ (i.e. $\Re$ over $k_{\mathrm{R}}$ ) is a rather small number on one side of the transition (of order $10^{-5}$ or less for $\lambda=1.5,2 k=1$ and $4 b=0.005$ ), while it is orders of magnitude larger on the other side $(\lambda=1.0$ and 0.5$)$.

To give an idea of what is going on in the transition from $\lambda=1.5$ to $\lambda=1.0$ and 0.5 (at $2 k=1$ and $4 b=0.005$ ) figs. 4 and 5 show the distribution of curvature (in units of the average lattice spacing) for $\lambda=1.5$ and $\lambda=0.5$, respectively. In the first case the distribution is well behaved and roughly gaussian. On the other hand in the second case long tails develop at both ends of the distribution indicating the presence of regions with very large positive curvature, and regions with very large negative one. (Also note the different vertical scales in the two cases.) For $\lambda=0.5$ and $\lambda=1.0$ the edge length distribution appears to be "sick". We have also tried to get the edge length distribution at $\lambda=0.5$ by starting from the one at $\lambda=1.5$ and 


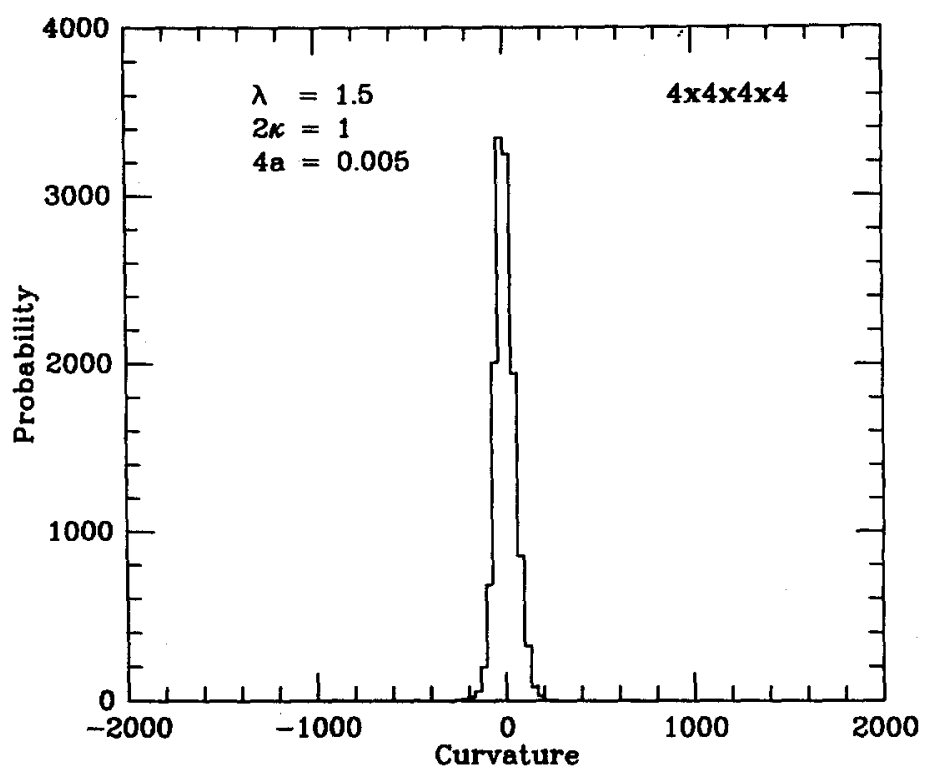

Fig. 4.

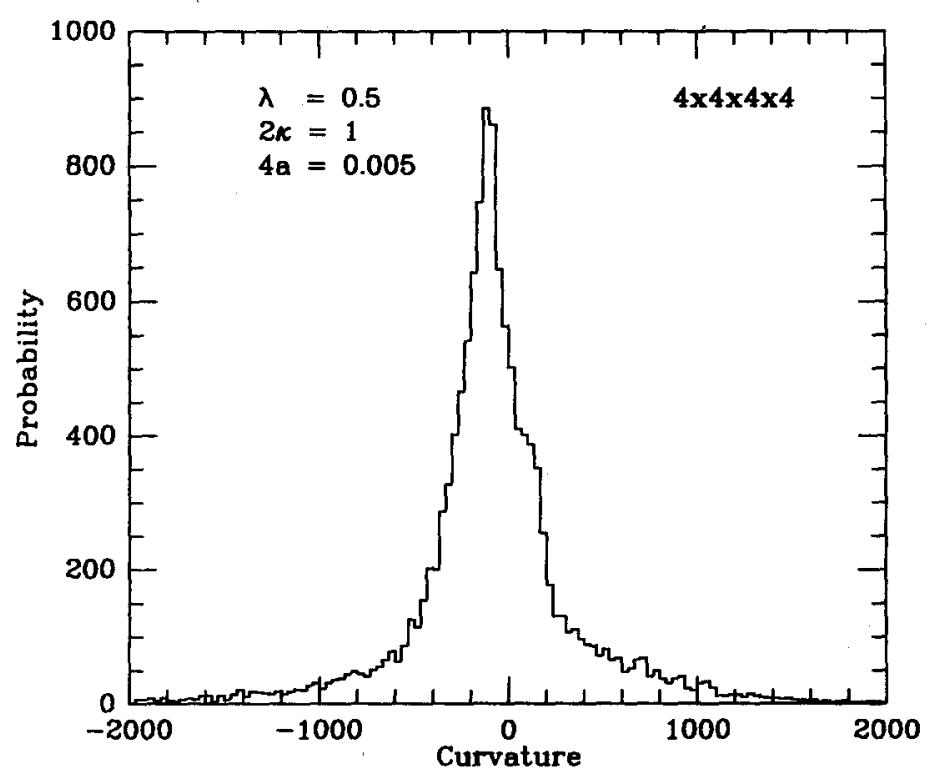

Fig. 5. 
re-thermalizing the lattice configuration. This was done on the $2^{4}$ lattice by decreasing $\lambda$ in steps of 0.1 and doing 100 iterations at each value. At $\lambda=0.5$ (therefore after a total of 1000 iterations) the edge length configuration looked rather similar to the one at $\lambda=1.5$, with a small positive average curvature. This fact presumably indicates the existence of long-lived metastable states. Once the edges get "locked in" in one configuration, it takes many iterations to move them out again.

Another important point concerns the question of unitarity. In lattice gravity the lattice spacing, and thus the ultraviolet cutoff, is a dynamically determined quantity, which depends on the values of the bare coupling constants. This situation is quite different from ordinary lattice field theories, where the lattice spacing is independent of the bare couplings. There violations of unitarity are common, but they always happen at energies close to the cutoff scale. It is thus quite plausible (and is supported by simple arguments) that in higher derivative lattice gravity the mass of the potentially dangerous spurious states is always above the (dynamical) ultraviolet cutoff. Unitarity can in principle be checked by looking at positivity in the decay of correlations like the one in equation (5.11). This is feasible, but has not been done yet.

The research of H.W.H. was supported by the US Department of Energy under grant no. DE-AC02-76ER02220. R.M.W. is grateful for hospitality at the Institute for Advanced Study.

\section{References}

[1] N.H. Barth and S.M. Christensen, Phys. Rev. D28 (1983) 1876

[2] S.W. Hawking, in General relativity - an Einstein centenary survey, ed. S.W. Hawking and W. Israel (CUP, 1979)

[3] G. 't Hooft and M. Veltman, Ann. Inst. Henri Poincaré 20 (1974) 69

[4] M. Veltman, in Methods in field theory, Les Houches Lecture notes session XXVIII (1975); G. 't Hooft, in Recent developments in gravitation, Cargèse Lecture notes (1978)

[5] K.G. Wilson and J. Kogut, Phys. Reports 12C (1974) 76;

K.G. Wilson, Rev. Mod. Phys. 55 (1983) 583;

H.B. Nielsen, in The constants of physics, Proc. Roy. Soc. of London (1983)

[6] B. DeWitt and R. Utiyama, J. Math. Phys. 3 (1962) 608;

R.P. Feynman, Acta Phys. Pol. 24 (1963) 697;

S. Weinberg, in General relativity - An Einstein centenary survey, ed. S.W. Hawking and W. Israel (CUP, 1979)

[7] K.S. Stelle, Phys. Rev. D16 (1977) 953

[8] J. Julve and M. Tonin, Nuovo Cim. 46B (1978) 137;

E.T. Tomboulis, Phys. Lett. 70B (1977) 361, 97B (1980) 77;

S. Adler, Rev. Mod. Phys. 54 (1982) 729

[9] E.S. Fradkin and A.A. Tseytlin, Phys. Lett. 104B (1981) 377; Nucl. Phys. B201 (1982) 469;

E.S. Fradkin and A.A. Tseytlin, Phys. Lett. 106B (1981) 63

[10] E.T. Tomboulis, Phys. Rev. Lett. 52 (1984) 1173;

E.T. Tomboulis, in Quantum theory of gravity: essays in honor of the 60th birthday of Bryce S.

DeWitt (Adam Hilgar, Bristol, 1983) 
[11] G. Parisi, in The 1984 Les Houches lectures on gauge theories;

H.W. Hamber, plenary talk at the VII International Congress on Mathematical Physics, Boulder, Colorado, 1983 (Physica 124A (1984) 365)

[12] T. Regge, Nuovo Cim. 19 (1961) 558

[13] C. Lanczos, Ann. of Math. 39 (1938) 842

[14] G.W. Gibbons and M.J. Perry, Nucl. Phys. B146 (1978) 90;

S.M. Christensen and M.J. Duff, Nucl. Phys. B170 [FS1] (1980) 480

[15] N.P. Konopleva and V.N. Popov, Gauge fields (Harwood Academic, New York, 1979)

[16] H.W. Hamber and R.M. Williams, Nucl. Phys. B248 (1984) 392

[17] H.W. Hamber, Simplicial quantum gravity, Les Houches Lecture notes, session XLIII (1984) (North-Holland), to be published

[18] S.I. Goldberg, Curvature and homology, ch. 3 (Dover, New York, 1962)

[19] S. Elitzur, private communication

[20] J. Cheeger, W. Müller and R. Shrader, Comm. Math. Phys. 92 (1984) 405

[21] M. Roček and R.M. Williams, Phys. Lett. 104B (1981) 31; Z. Phys. C21 (1984) 371

[22] J.B. Hartle, Simplicial minisuperspace I: general discussion, UC Santa Barbara preprint (June 1984) J. Math. Phys., to be published;

J.B. Hartle, Simplicial minisuperspace II: some classical solutions on simple triangulations, UCSB preprint TH-68, to be published in J. Math. Phys.

[23] N. Christ, R. Friedberg and T.D. Lee, Nucl. Phys. B202 (1982) 89;

C. Itzykson, Cargèse Lecture Notes 1983, to be published

[24] H. Coxeter, Regular polytopes (Methuen, London, 1948)

[25] J. Frölich, Regge calculus and discretized gravitational functional integrals, IHES preprint (1981) unpublished

[26] K. Osterwalder and R. Schrader, Comm. Math. Phys. 31 (1973) 83, 42 (1975) 281;

D. Brydges, J. Frölich and E. Seiler, Ann. of Phys. (NY) 121 (1979) 227

[27] H.W. Hamber and R.M. Williams, Nonperturbative simplicial quantum gravity, IAS preprint (March 1985) Phys. Lett. B, to be published

[28] G. Parisi and Y.-S. Wu, Sci. Sinica 24 (1981) 483;

J.R. Klauder, Stochastic quantization, Lectures given at the XXII Schladming school, Acta. Phys. Austr. Suppl. XXV (1983) 251

[29] G. Parisi, Nucl. Phys. B180 [FS2] (1981) 378, B205 [FS5] (1982) 337

[30] T. Fukai and K. Okano, Waseda preprint WU-HEP-84-8

[31] H.W. Hamber and R.M. Williams, Two-dimensional simplicial quantum gravity, IAS preprint (May 1985)

[32] S.W. Hawking, Phys. Lett. 134B (1984) 403 